\title{
Tissue Attenuation Estimation by Mean Frequency Downshift and Bandwidth Limitation
}

\author{
Ziemowit Klimonda, Michiel Postema, Senior Member, IEEE, Andrzej Nowicki, and Jerzy Litniewski,
}

\begin{abstract}
Attenuation of ultrasound in tissue can be estimated from the propagating pulse center frequency downshift. This method assumes that the envelope of the emitted pulse can be approximated by a Gaussian function and that the attenuation depends linearly on frequency. The resulting downshift of the mean frequency depends not only on attenuation, but also on pulse bandwidth and propagation distance. This kind of approach is valid for narrowband pulses and shallow penetration depth. However, for short pulses and deep penetration, the frequency downshift is rather large and the received spectra are modified by the limited bandwidth of the receiving system. In the paper the modified formula modelling the mean frequency of backscattered echoes is presented. The equation takes into account the limitation of the bandwidth due to band-pass filtration of the received echoes. This approach was applied to simulate the variation of the mean frequency of the pulse propagating for both: weakly and strongly attenuating media and for narrowband and wideband pulses. The behavior of both the standard and modified estimates of attenuation has been validated using RF data from a tissuemimicking phantom. The ultrasound attenuation of the phantom, determined with a corrected equation, was close to its true value, while the result obtained using the original formula was lower by as much as $50 \%$ at a depth of $8 \mathrm{~cm}$.
\end{abstract}

Index Terms-Tissue attenuation, frequency downshift, bandwidth limitation

\section{INTRODUCTION}

$\mathbf{T}$ ISSUE attenuation can characterize soft tissue, although natural biological variability limits its effectiveness. Proper knowledge of tissue attenuation is required to reduce shadowing artefacts [1] or determination of scatterer concentration and statistical parameters of the backscatter [2]. Numerous reports suggest the relationship between attenuation and the pathological state of tissue. It has been shown, that the slope of the attenuation coefficient, combined with the statistical parameters of the image texture, can be used to diagnose diffuse liver disease [3]. Attenuation values can characterize cirrhotic and fatty human liver [4] and breast tumors [5], but also help to discriminate between cancerous and healthy tissue [6]. Changes of attenuation in the cervix have been helpful in prediction of premature delivery in rats [7] and in humans [8]-[11]. Potentially it can be used for skin lesion classification [12]. It has been shown that attenuation changes are related to the degeneration of bovine articular cartilage [13], thermal coagulation [14] and HIFU treatment [15], [16].

Several approaches to attenuation estimation have been described, such as spectral shift [17]-[19], spectral difference [20]-[22], a reference phantom method [23], and a hybrid spectral domain method [24]. An extensive overview of spectral methods is presented in [25] and [26].
The spectral shift method exploits the downshift of the center frequency of the ultrasound pulse propagating in an attenuating medium. This downshift is caused by the frequencydependence of attenuation and can be used to determine the attenuation coefficient.

With the spectral difference method, the attenuation is found using the logarithmic spectral difference of power spectra. These spectra are calculated from different segments of received ultrasound signals corresponding to different depths of the medium.

With the reference phantom method, a well-specified, reference phantom is used to correct the ultrasound system and diffraction effects. The power spectra of signals acquired from the examined area of the tissue sample are divided by corresponding spectra acquired from the reference phantom. The local attenuation is estimated from the changes of such normalized spectra with depth.

The hybrid spectral domain method combines the spectral difference and spectral shift methods. The spectra from samples are divided by corresponding reference spectra, corrected for the attenuation in a reference phantom and multiplied by a Gaussian function. The attenuation is estimated from the downshift of such corrected spectra.

In this study we concentrate on the spectral shift method. Generally with frequency downshift, the linear dependence between attenuation and frequency is assumed. A further assumption is a Gaussian spectrum of the interrogating pulse. With these conditions a linear equation can be derived linking the shift of the center frequency with an attenuation coefficient, the trasmited pulse bandwidth, and the propagation distance. However, there are many other factors which might cause a frequency shift of the received signals and render the above assumptions inappropriate. The variations in frequencydependence of backscatter is one of those factors [1], but considered negligible in the present work.

Furthermore, differences between real and reference values of sound speed in a medium may introduce a bias in the attenuation estimate [27]. Other factors include windowing and electronic noise affecting the attenuation estimate [28], [29], and beam diffraction causing the amplitudes of low frequency components to decrease as the depth of pulse propagation increases [30]. Furthermore, the received signals always have a limited frequency bandwidth due to band-pass filtration of the ultrasonic system itself, or of the subsequent processing system, deforming the frequency spectra of received signals. The more of the received pulse is out-of-band, the greater is the deformation of the spectrum and the greater is the error in the attenuation estimate. 
In this paper we propose a novel algorithm for attenuation estimation from the mean frequency downshift. This modified algorithm inteds to reduce the estimation errors resulting from the noise component and the beam diffraction. Our approach helps to reduce the impact of these factors by proper bandpass filtration. In other words, the mean frequency is estimated only from the part of the spectrum where the distortions are relatively small. Thus, the linear model of mean frequency downshift is modified by taking into account the changes of the signal spectrum due to band-pass filtration. In 2012, we reported the qualitative validation of the method originally proposed [31]. The following work presents the theoretical background of the method and the results of its experimental validation. In Section II, the impact of the noise and of the beam diffraction on the signal mean frequency estimate are discussed. The equation describing the mean frequency of the filtered Gaussian spectrum is derived in Section III. The verification of the equation using simulated data is presented in Section IV. In Section V, experimental data is subjected to the new algorithm. The results are compared with non-modified attenuation estimates.

\section{NOISE AND BEAM DIFFRACTION EFFECTS ON SPECTRAL MEAN FREQUENCY}

\section{A. Effect of noise}

When the amplitude of a propagating pulse decreases due to attenuation, the signal-to-noise ratio (SNR) also drops. For a low SNR, the noise frequency component is relatively high and the combined spectrum of the received signal (signal and noise) is shifted towards the noise mean frequency [29]. This is also the case with signals from large depth. The signal spectrum is downshifted due to attenuation but the noise component increases the received signal mean frequency. Accordingly, the mean frequency estimate is overestimated and the overestimation increases with SNR decrease.

Figure 1 demonstrates an ilustrative example of normalized spectra from signals backscattered in a tissue-mimicking phantom at a $1-\mathrm{cm}$ (black line) and 8 - $\mathrm{cm}$ depth (gray line). The spectral noise level is indicated by arrow. In the case

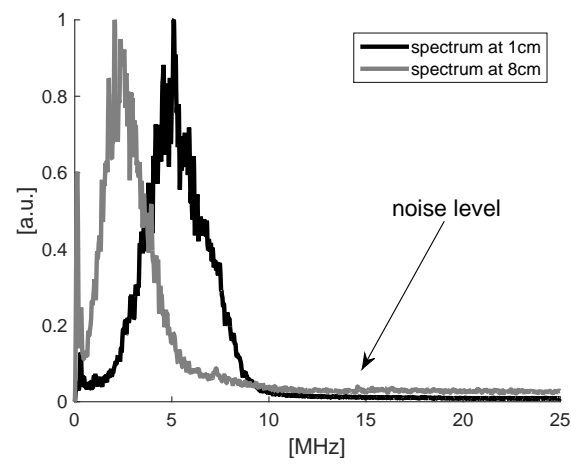

Fig. 1. Normalized spectra of the signal acquired from A tissue-mimicking phantom at $1-\mathrm{cm}$ and $8-\mathrm{cm}$ depths.

of $8-\mathrm{cm}$ penetration depth, the mean frequency calculated in the $0-25 \mathrm{MHz}$ frequency range equals $5.0 \mathrm{MHz}$, while after low-pass filtering with a cut-off frequency of $8 \mathrm{MHz}$, the mean frequency dropped to $2.9 \mathrm{MHz}$, corresponding to the peak frequency of the spectrum (gray line). Figure 1 also shows some distortions for frequency between 0 and $0.6 \mathrm{MHz}$. The source of these distortions is attributed to the receiving system. Thus, there is a part of the signal spectrum which clearly does not fit into the Gaussian pulse model. Therefore, bandpass filtration should be used to isolate signal from noise. Bandpass filtration is a standard method used in medical ultrasound techniques to increase the SNR or to isolate the desired frequency range of a received signal [32].

\section{B. The effect of beam diffraction}

Beam diffraction is an important factor affecting the ultrasonic wave propagation. The diffraction correction $D_{\mathrm{L}}$ for a piston source is given by [33]

$$
D_{L}=1-e^{-i(2 \pi / s)}\left[J_{0}(2 \pi / s)+i J_{1}(2 \pi / s)\right]
$$

where $J_{0}$ and $J_{1}$ are the zeroth and first order Bessel functions of the first kind, $s=\Delta x c /\left(f a^{2}\right)$, in which $c$ is a speed of sound and $a$ is transducer radius. The diffraction correction is defined as a normalized integral of the acoustic field over the circle with the surface equal to the surface of the piston source and located coaxially at a distance $\Delta x$. The integral is normalized by a similar integral of the plane wave acoustic field [33]. It describes how the beam diffraction affects the received signal comparing to the plane wave. Figure 2 shows the absolute value of the $D_{\mathrm{L}}$ as a function of the frequency, at a depth of $1 \mathrm{~cm}$ and $8 \mathrm{~cm}$. Both plots were calculated for a transducer radius equal to $7.5 \mathrm{~mm}$ used in experiments and described further in Section V. Figure 2 shows, that the

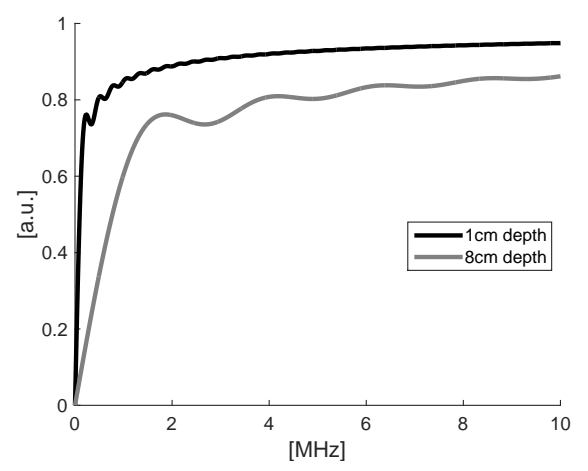

Fig. 2. The absolute values of diffraction correction $\left|D_{\mathrm{L}}\right|$ for a piston source with radius $7.5 \mathrm{~mm}$, calculated for depths of $1 \mathrm{~cm}$ and $8 \mathrm{~cm}$.

diffraction mostly distorts the lower part of the spectrum. In our case, the part of the spectrum below $2 \mathrm{MHz}$ is strongly affected by the beam diffraction, while for the higher frequencies, the distortion is substantially smaller. The pulse spectrum is moved to the low frequency range where the correction for diffraction effects are especially significant, particularly in the case of deep penetration, due to the pulse mean frequency downshift caused by attenuation.

In short, noise and beam diffraction affects the mean frequency estimate. This impact could be limited by considering 
only that part of the received signal spectrum, where the distortions are small. It can be done by band-pass filtering prior to the mean frequency estimation. However, such filtering modifies the signal spectrum whilst the Gaussian pulse model is no longer valid. Thus, the model should be modified to take into account the band-pass filtration of the received signal and the resulted distortion of its spectrum. The new equation allows for the recovery of the Gaussian pulse mean frequency when the ultrasonic backscatter is band-pass filtered, which is described in the next section.

\section{Summary OF ALgORITHM}

Let us assume a Gaussian pulse with carrier frequency $f_{0}$ propagating through the attenuating medium and described by the following equation

$$
p(t)=\sin \left(2 \pi f_{0} t\right) e^{\left(-\frac{t^{2}}{2 \sigma_{t}^{2}}\right)},
$$

where $t$ is time and $\sigma_{t}^{2}$ is variance of the Gaussian envelope. The Fourier amplitude spectrum is

$$
P(f)=\frac{\sigma_{t}}{2 \sqrt{2 \pi}}\left|e^{\left[-\frac{\left(f-f_{0}\right)^{2}}{2\left(\frac{1}{2 \pi \sigma_{t}}\right)^{2}}\right]}-e^{\left[-\frac{\left(f+f_{0}\right)^{2}}{2\left(\frac{1}{2 \pi \sigma_{t}}\right)^{2}}\right]}\right| .
$$

Thus, the amplitude spectrum of $p(t)$ is even and has the form of absolute value of the difference of two Gaussian functions with peaks at $f_{0}$ and $-f_{0}$, respectively. For $f_{0} \gg 0$, the one sided spectrum can be approximated by a single Gaussian function with a peak at $f_{0}$. Assuming that the attenuation varies linearly with frequency $f$, the spectrum of the propagating pulse is a product of the Gaussian function and the exponential function representing the attenuation. Thus, the one sided power spectrum $S(f)$ of $p(t)$ can be approximated as follows

$$
S(f)=A e^{\left[-\frac{\left(f-f_{0}\right)^{2}}{\sigma^{2}}\right]} e^{(-2 \alpha f \Delta x)},
$$

where $f_{0}$ corresponds to the spectral peak of the transmitted pulse, $\sigma^{2}=1 /\left(2 \pi \sigma_{t}\right)^{2}$ is the variance of the amplitude spectrum, $\alpha$ is the attenuation coefficient, $\Delta x$ is the distance traveled by scanning pulse and $A=\sigma_{t}^{2} / 8 \pi^{2}$ is a scaling factor. Expression (4) can be transformed as follows:

$$
\begin{aligned}
& S(f)=A e^{\left[-\frac{f^{2}+\left(2 \sigma^{2} \alpha \Delta x-2 f_{0}\right) f+f_{0}^{2}}{\sigma^{2}}\right]}= \\
& =A e^{\left(\sigma^{2} \alpha^{2} \Delta x^{2}-2 \alpha \Delta x f_{0}\right)} e^{\left[-\left(\frac{f-f_{\mathrm{p}}}{\sigma}\right)^{2}\right]} .
\end{aligned}
$$

Expression (5) shows that the power spectrum of the scanning pulse traveling in linear attenuating medium is given by a Gaussian function, but with a new peak frequency $f_{\mathrm{p}}$ instead of $f_{0}$. Peak frequency $f_{\mathrm{p}}$ is given by expression (6)

$$
f_{\mathrm{p}}=f_{0}-\sigma^{2} \alpha \Delta x \text {. }
$$

This common expression is often used in the spectral shift method [26]. In this case, the local attenuation coefficient $\alpha$ is equal to [34]:

$$
\alpha=-\frac{1}{\sigma^{2}} \frac{\Delta f_{\mathrm{p}}}{\Delta x} \underset{\Delta x \rightarrow 0}{\rightarrow}-\frac{1}{\sigma^{2}} \frac{d f_{\mathrm{p}}}{d x} .
$$

For a Gaussian pulse, the frequency of the spectral peak $f_{\mathrm{p}}$ is close to the pulse mean frequency $f_{\mathrm{m}}$. Thus, in this case $f_{\mathrm{m}}$ can be used as an estimate of $f_{\mathrm{p}}$ and replace it in (7) in order to estimate the attenuation coefficient which is convenient as the mean frequency $f_{\mathrm{m}}$ can be readily estimated from the ultrasound data.

$$
f_{\mathrm{m}} \approx f_{\mathrm{p}}
$$

However, approximation (8) is not always appropriate. The basic idea is graphically explained in Figure 3 for arbitrary Gaussian spectra and two filter bandwidths, one wide and the other narrow.

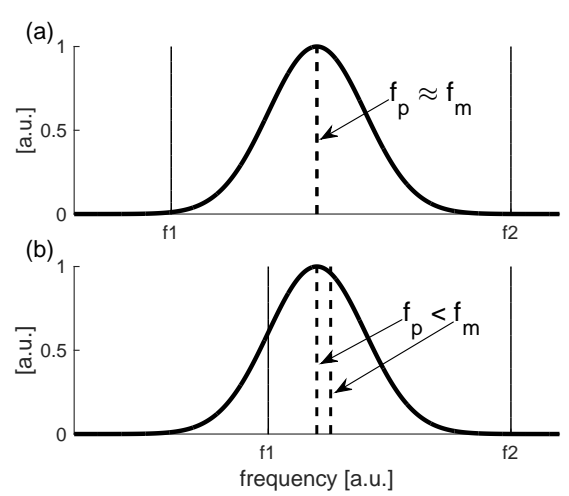

Fig. 3. The difference between the peak and mean frequencies of a virtual Gaussian spectrum computed assuming (a) a wide and (b) a narrow filter bandwidth.

Assuming that the received signal is band-pass filtered with the cut-off frequencies $f_{1}$ and $f_{2}$, approximation (8) is appropriate only if the received pulse spectrum is inside the filter bandwidth (Fig. 3). It is not valid if a significant amount of spectral energy is outside of the filter bandwidth (Fig. 3b) yielding an incorrect attenuation estimation.

Assuming that the pulse is perfectly reflected and subsequently detected by the receiving system which introduces band-pass filtration, the mean frequency can be determined using [32]

$$
f_{\mathrm{m}}=\frac{\int_{0}^{\infty} f S(f)}{\int_{0}^{\infty} S(f)} .
$$

Assuming that the band-pass filtration can be described by ideal filter with a rectangular transfer function with cut-off frequencies $f_{1}$ and $f_{2}$, the mean frequency $f_{\mathrm{m}}$ is given by (10)

$$
f_{\mathrm{m}}=\frac{\int_{f_{1}}^{f_{2}} f S(f)}{\int_{f_{1}}^{f_{2}} S(f)},
$$

where the denominator can be expressed by

$$
\begin{gathered}
\int_{f_{1}}^{f_{2}} S(f) d f=B \int_{f_{1}}^{f_{2}} e^{\left[-\left(\frac{f-f_{\mathrm{p}}}{\sigma}\right)^{2}\right]} d f \\
=B \frac{\sqrt{\pi}}{2} \sigma\left[\operatorname{erf}\left(\frac{f_{2}-f_{\mathrm{p}}}{\sigma}\right)-\operatorname{erf}\left(\frac{f_{1}-f_{\mathrm{p}}}{\sigma}\right)\right],
\end{gathered}
$$

where $\operatorname{erf}()$ is the error function and $B$ is given by (12)

$$
B=A e^{\sigma^{2} \alpha^{2} \Delta x^{2}-2 \alpha \Delta x f_{0}} .
$$


The numerator of (10) can be transformed as follows

$$
\begin{aligned}
\int_{f_{1}}^{f_{2}} f S(f) \mathrm{d} f= & B \int_{f_{1}}^{f_{2}} f e^{-\left(\frac{f-f_{\mathrm{p}}}{\sigma}\right)^{2}} \mathrm{~d} f \\
= & B\left(\int_{t_{1}}^{t_{2}} \sigma^{2} t e^{-t^{2}} \mathrm{~d} t+f_{\mathrm{p}} \int_{t_{1}}^{t_{2}} \sigma e^{-t^{2}} \mathrm{~d} t\right) \\
= & B \frac{\sigma^{2}}{2}\left[e^{-\left(\frac{f_{1}-f_{\mathrm{p}}}{\sigma}\right)^{2}}-e^{-\left(\frac{f_{2}-f_{\mathrm{p}}}{\sigma}\right)^{2}}\right] \\
& +B f_{\mathrm{p}} \frac{\sqrt{\pi}}{2} \sigma\left[\operatorname{erf}\left(\frac{f_{2}-f_{\mathrm{p}}}{\sigma}\right)\right. \\
& \left.-\operatorname{erf}\left(\frac{f_{1}-f_{\mathrm{p}}}{\sigma}\right)\right] .
\end{aligned}
$$

Finally, the $f_{\mathrm{m}}$ is given by

$$
f_{\mathrm{m}}=f_{\mathrm{p}}-\frac{\sigma}{\sqrt{\pi}} \frac{e^{\left[-\left(\frac{f_{2}-f_{\mathrm{p}}}{\sigma}\right)^{2}\right]}-e^{\left[-\left(\frac{f_{1}-f_{\mathrm{p}}}{\sigma}\right)^{2}\right]}}{\operatorname{erf}\left(\frac{f_{2}-f_{\mathrm{p}}}{\sigma}\right)-\operatorname{erf}\left(\frac{f_{1}-f_{\mathrm{p}}}{\sigma}\right)}
$$

where the $f_{\mathrm{p}}$ is given by equation (6). It is clear that the pulse mean frequency differs from the frequency of the spectral peak frequency. The expression describing the measured mean frequency $f_{\mathrm{m}}$ is the sum of the frequency $f_{\mathrm{p}}$ and the bias component which is a function of $f_{\mathrm{p}}$. If $f_{1}$ approaches $-\infty$ and $f_{2}$ approaches $\infty$, the bias component disappears and $f_{\mathrm{m}}=f_{\mathrm{p}}$. Nevertheless, the frequency $f_{1}$ is always greater than or equal to zero and $f_{2}$ is finite for pulses received by real system. Therefore the mean frequency is only an approximation of the peak frequency.

Figure 4 shows the function $f_{\mathrm{m}}\left(f_{\mathrm{p}}\right)$ calculated for three different $\sigma$ parameters. We have empirically verified that equation (14) gives unique and stable solutions for the values of $f_{\mathrm{p}}$ corresponding with the attenuation values occurring in soft tissue, frequencies up to $15 \mathrm{MHz}$ and pulse bandwidth up to $90 \%$.

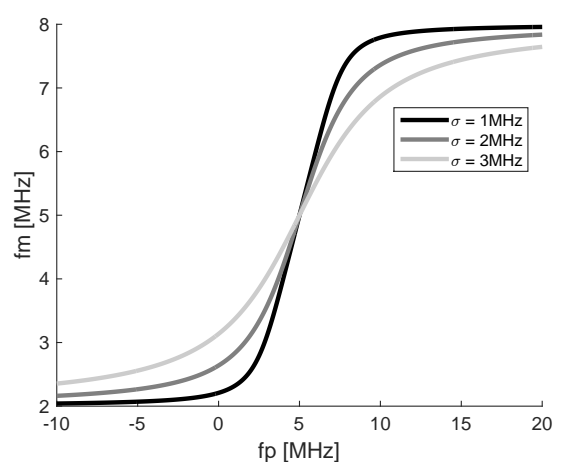

Fig. 4. The function $f_{\mathrm{m}}\left(f_{\mathrm{p}}\right)$ calculated for three different $\sigma$ parameters of the Gaussian pulse. The cut-off frequencies $f_{1}$ and $f_{2}$ were 2 and $8 \mathrm{MHz}$, respectively.

To determine the attenuation coefficient from band-pass filtrated data using the estimated $f_{\mathrm{m}}$ and equation (7), the true peak frequency $f_{\mathrm{p}}$ should first be calculated. Peak frequency $f_{\mathrm{p}}$ is an implicit function of $f_{\mathrm{m}}$. Therefore it can be calculated by numerically solving equation (14). Then, the attenuation can be estimated from $f_{\mathrm{p}}$ using (7). In the following section the results of simulations of (14) are presented.

\section{COMPuter Simulations}

\section{A. Simulation procedure}

Equation (14) describes the mean frequency changes for band-pass filtrated backscattered signals. It was validated using simulated RF data. For simulations of the signal received at the transducer a simple, 1-dimensional $(1 D)$ geometry model of tissue and incidence of plane wave was assumed. The $1 D$ model was applied as a direct consequence of the $180^{\circ}$ backscattering assumption. It was also assumed that the attenuation is the only factor influencing the mean frequency shift. It is important to emphasize here that the only reason for the development of a simulation model was to test whether (14) correctly predicts the mean frequency down shift calculated from the band-filtered backscatter. The final validation of the method proposed including filtering of the noise and diffraction distortions was performed in phantom experiments. The backscattered signal from tissue was simulated as follows. A set of point scatterers was uniformly distributed along the $Z$ axis up to a depth of $10 \mathrm{~cm}$ in a medium attenuating linearly with frequency. The average number of scatterers per wavelength equaled 53 as discussed in [35], [37]. The speed of sound in the simulated medium was $1540 \mathrm{~m} / \mathrm{s}$. The scatterers reflectivity was also uniformly randomly distributed. Each scatterer was considered a secondary sound source. The transmit pulse had a Gaussian envelope and $5.6 \mathrm{MHz}$ carrier frequency and a $50 \%$ or a $76 \%$ bandwidth $(-6 \mathrm{~dB})$. The transducer transfer function was identical to the transmit pulse amplitude spectrum. The backscattered echoes were simulated by superposition of all of the elementary, individually scattered wavelets, taking into account the transducer transfer function, phase differences caused by spatial location of individual scatterer and attenuation. The tissue attenuation coefficients $\alpha=0.5$ and $1.5 \mathrm{~dB} /(\mathrm{cm} \cdot \mathrm{MHz}))$ were chosen in subsequent simulations. $33 \mathrm{RF}$ lines were simulated for each value of attenuation coefficient. The band-pass filtering was performed using the fourth order Butterworth filters with passband 1$-12 \mathrm{MHz}$ and $3-7 \mathrm{MHz}$. The variation of mean frequency $f_{\mathrm{m}}$ along the penetration path was estimated from filtrated RF lines by means of I/Q algorithm [36], [37] taking $10 \mathrm{~mm}$ long sample window. Next, the average from 33 mean frequency $f_{\mathrm{m}}$ lines was calculated and compared with the mean frequency predicted from (8) and (14).

\section{B. Simulation results and discussion}

The results of the simulations are presented in Figures 5 7. The $f_{\mathrm{m}}$ lines (gray) were estimated from simulated echoes while the predictions of the linear (8) and the new equation (14) are represented by black dashed line and solid lines, respectively.

The results for the medium attenuation of $0.5 \mathrm{~dB} /(\mathrm{cm} \cdot \mathrm{MHz}))$ and a $50 \%$ pulse bandwidth are presented in Figure 5. For wideband filtration (Fig. 5a), there are no visible differences between predictions of both equations. For narrowband filtration (Fig. 5b), some small differences are visible and the corrected equation fits a slightly better to the estimate. 


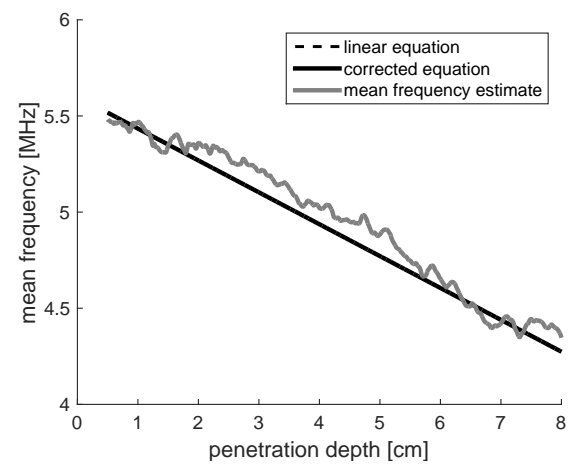

(a)

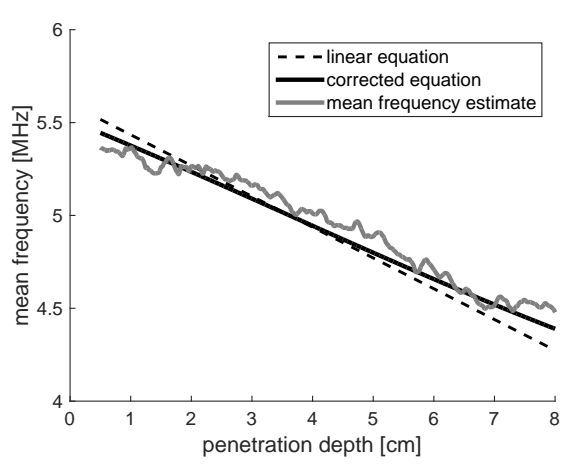

(b)

Fig. 5. Mean frequency estimate and predictions of both equations - linear and corrected. The RF data were simulated. Medium with attenuation coefficient equal $0.5 \mathrm{~dB} /(\mathrm{cm} \cdot \mathrm{MHz})$ and $5.6 \mathrm{MHz}$ pulse with $50 \%$ bandwidth was assumed. The filter passband was equal to (a) $1-12 \mathrm{MHz}$ and (b) $3-7 \mathrm{MHz}$.

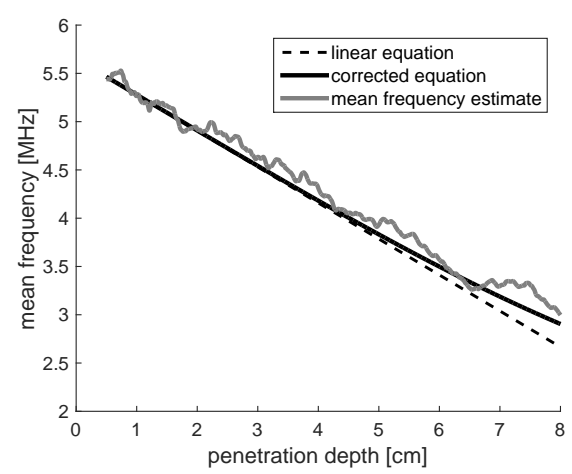

(a)

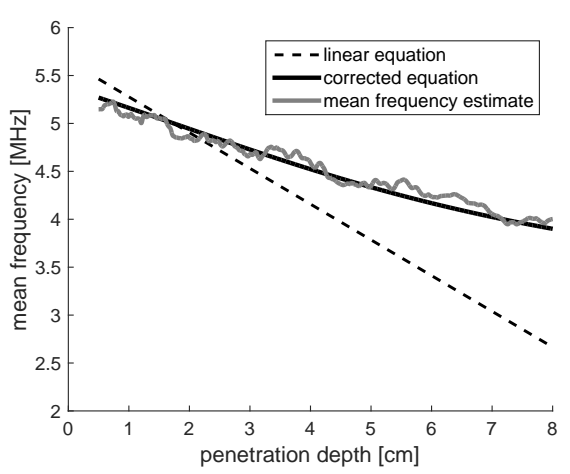

(b)

Fig. 6. Mean frequency estimate and predictions of both equations - linear and corrected. The RF data were simulated. Medium with attenuation coefficient equal $0.5 \mathrm{~dB} /(\mathrm{cm} \cdot \mathrm{MHz})$ and $5.6 \mathrm{MHz}$ pulse with $76 \%$ bandwidth was assumed. The filter passband was equal to (a) $1-12 \mathrm{MHz}$ and (b) $3-7 \mathrm{MHz}$.

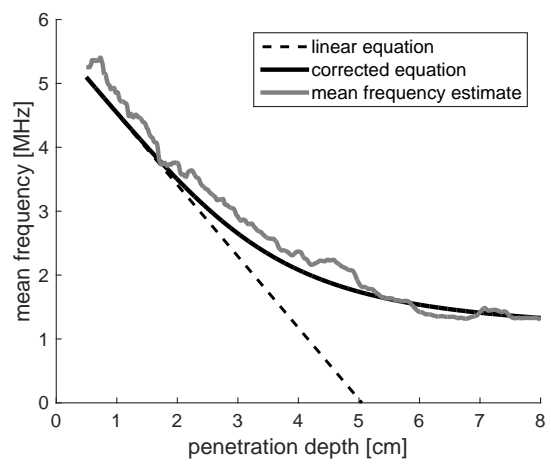

(a)

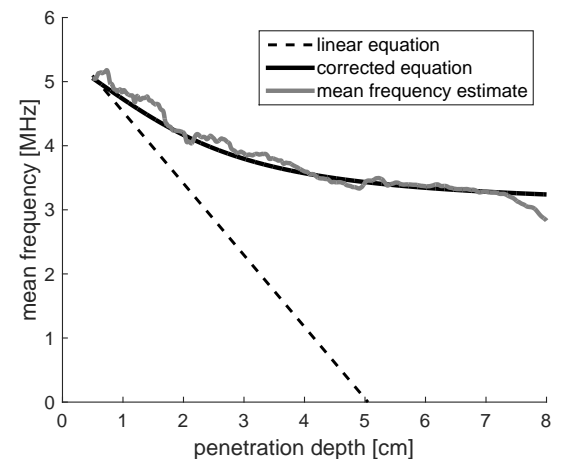

(b)

Fig. 7. Mean frequency estimate and predictions of both equations - linear and corrected. The RF data were simulated. Medium with attenuation coefficient equal $1.5 \mathrm{~dB} /(\mathrm{cm} \cdot \mathrm{MHz})$ and $5.6 \mathrm{MHz}$ pulse with $76 \%$ bandwidth was assumed. The filter passband was equal to (a) $1-12 \mathrm{MHz}$ and (b) $3-7 M H z$.

The estimates of mean frequencies for the medium attenuation of $0.5 \mathrm{~dB} /(\mathrm{cm} \cdot \mathrm{MHz})$ and pulses of $76 \%$ bandwidth are presented in Figure 6. Here, the use of wideband filtration (Fig. 6a) results in very small differences between predictions of both equations, as previously (Fig. 5a). However, the difference between the old and the new approach is clearly visible for narrowband filtration (Fig. 6b), where at the distance of $8 \mathrm{~cm}$ the difference exceeds $1.2 \mathrm{MHz}$ and modified equation predicts correctly mean frequency of simulated echoes.

The downshifts of mean frequency in highly attenuating medium $(1.5 \mathrm{~dB} /(\mathrm{cm} \cdot \mathrm{MHz}))$ for pulses of $76 \%$ bandwidth are shown in Figures $7 \mathrm{a}$ and $7 \mathrm{~b}$. In this case the differences between old and new equations are clearly visible even for wideband filtration. The corrected equation fits the estimated mean frequency well along the whole penetration depth, while the linear equation fits the $f_{\mathrm{m}}$ line only for wideband filtration 
and a depth of less than $2 \mathrm{~cm}$ (Fig. 7a).

Presented results indicate that equation (14) correctly describes the $f_{\mathrm{m}}$ shift caused by attenuation even for the narrow band filtering of the simulated echoes.

\section{Phantom Experiment}

\section{A. Measurement setup}

The experimental setup consisted of a flat $15 \mathrm{~mm}$ diameter transducer, $5.6 \mathrm{MHz}$ center frequency, (IMASONIC ME5 - $1 / 2^{\prime \prime}$, France), a pulser-receiver (Panametric 5900 PR) and a sampling oscilloscope (Agilent Infinium 54810A). First, the pulse spectrum in water was examined to measure the transmitted pulse bandwidth and then calculate an estimate of standard variation $\sigma$ in (8) and (14). The transducer was driven by short pulse and the ultrasound pulse reflected from acoustic reflector placed in water at $60 \mathrm{~mm}$ depth was recorded. The amplitude spectrum of the pulse was calculated and Full Width at Half Maximum (FWHM) was determined. Then the standard deviation $\sigma$ was calculated using the relation $\mathrm{FWHM} \approx 2.35 \sigma$. The standard deviation was equal $1.68 \mathrm{MHz}$ and was used as an estimate of $\sigma$ in equations (8) and (14). $33 \mathrm{RF}$ backscattered echoes from the tissue mimicking phantom (Dansk Phantom Service, model 1054, attenuation coefficient equal $0.7 \mathrm{~dB} /(\mathrm{cm} \cdot \mathrm{MHz})$ ) were recorded. Each echo was acquired in different phantom location assuring statistical independence of ultrasonic data. The measured RF data were band-pass $(2-8 \mathrm{MHz})$ filtered, to limit the impact of the beam diffraction and presence of noise on the final attenuation estimate. The cut-off frequencies were chosen basing on the plots of the backscattered spectra (Fig. 1) and diffraction correction $D_{\mathrm{L}}$ (Fig. 2). The lower cut-off frequency was chosen to remove the part of the signal spectrum which is the most distorted by beam diffraction. The higher cutoff frequency was chosen to increase the SNR especially in signals from greater depths. As a reference, the measured RF data without initial band-pass filtering were processed in the same way. The mean frequency variation with the depth was estimated using exactly the same procedures as in the case of simulated echoes i.e. using the I/Q algorithm with a window length corresponding to a $1 \mathrm{~cm}$ depth. Data from a depth of up to $0.5 \mathrm{~cm}$ were not taken into account because of the strong and multiple reflections from the surface of the phantom. The resulting 33 mean frequency lines were averaged and compared with the models given by (8) and (14). The attenuation distribution was estimated as follows. Each mean frequency line was smoothed using the Singular Spectrum Analysis (SSA) algorithm [40]. Next, the attenuation was estimated using equations (7), (8) (linear equation) and (14) (corrected equation). Equation (14) was numerically solved for $f_{p}$ using Matlab ${ }^{\circledR}$ (The MathWorks, Ltd., Natick, MA) function fzero(). Finally, the attenuation lines were averaged and the standard error of the mean was calculated for each point of the averaged attenuation line.

\section{B. Measurement results and discussion}

The reference plot of the mean frequency changes estimated without the initial band-pass filtration of RF data are shown in
Figure 8 (gray line). The predictions from both equations for $f_{\mathrm{m}}$ (linear 8 and corrected 14) are plotted by dashed and solid black lines respectively. For this plot the cut-off frequencies $f_{1}$ and $f_{2}$ in 14 equaled 0 and $25 \mathrm{MHz}$ respectively, where the upper band corresponds to sampling frequency $(50 \mathrm{MHz})$. For low penetration depth both models predict similar drop

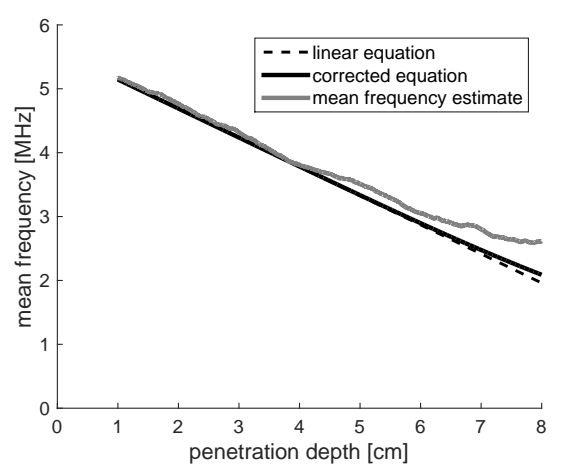

Fig. 8. Mean frequency estimate and predictions of both equations - linear and corrected. The RF data were acquired from tissue-mimicking phantom and were processed without initial filtration.

of the mean frequency that agrees well with the estimated mean frequency line. For larger depths the estimated values are clearly higher and the difference between the estimated and both predicted lines increases with penetration depth. The frequency range of filtration band is $(0-25 \mathrm{MHz})$ which can be considered large, thus both approaches operate very similarly. The corrected method does not cut out the low frequency components of the signal spectrum responsible for the diffraction distortion. The upper limit of filtration is too high to limit the influence of noise. Consequently, the frequency line calculated using the corrected equation is not consistent with the mean frequency estimate.

The mean frequency curves estimated from the band-pass filtered $(2-8 \mathrm{MHz})$ echoes together with the frequency values predicted by (14) are presented in Figure 9. The comparison

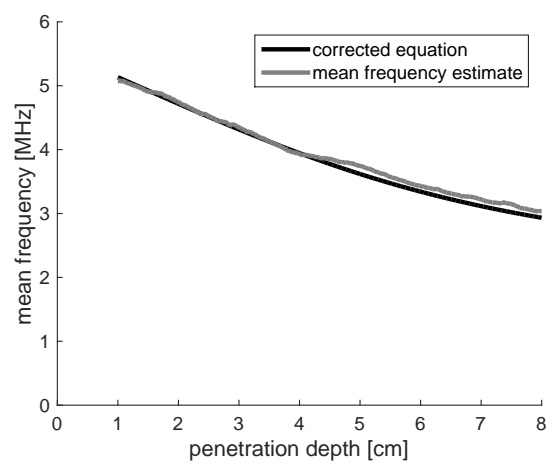

Fig. 9. Mean frequency estimate and prediction of the corrected equation. The RF data were initially processed using band-pass $(2-8 \mathrm{MHz})$ filter.

of the Figures 8 and 9 indicates, that band-pass filtration have clearly limited the difference between the mean frequency estimate and the prediction of the new model.

The plots of attenuation distribution corresponding to measured mean frequency data are shown in Figure 10. The attenu- 
ation values from non-filtered data were estimated using linear equation (8), while the (14) was used together with bandpass filtered data. The results obtained by the new method are close to the nominal phantom attenuation. At the same time the use of the equation (8) on the non-filtered signals leads to underestimation of the attenuation values on large depths. Although the standard error of the new estimate (gray

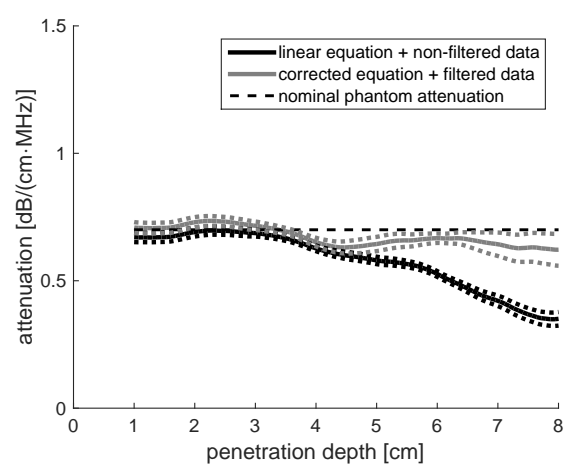

Fig. 10. Average attenuation distribution estimated using linear and non-linear equation. Dotted lines indicate the standard error of the mean. The RF data processed using the corrected equation were initially filtered using band-pass $(2-8 \mathrm{MHz})$ filter.

dotted lines) increases at larger depths compared to the error of the non-modified estimate (black dotted lines), it is still more accurate than the estimate obtained using non-modified method. The presented plots indicate that the estimate errors caused by the beam diffraction and the noise component can be reduced by proper band-bass filtration and use of the nonlinear model (eq. (14)) of the mean frequency drop.

However, the presented method have some limitations. The method assumes the propagation of a Gaussian pulse. Therefore, it will be not appropriate, when the shape of the interrogating pulse is far from Gaussian. For example, such situation occures in coded transmission technique. Moreover, the assymptotic behavior of the function $f_{m}\left(f_{p}\right)$ (Fig. 4) indicates, that for large frequency shift the small inaccuracy in $f_{m}$ estimation results in large error in $f_{p}$. This is the probable reason of the increase of the standard error in the new estimate (Fig. 10, gray dotted lines). Finally, this method require more calculations compared to non-modified frequency shift method, thus it is computationally more expensive.

Additionally, we used the I/Q algorithm in order to estimate the mean frequency of the signal. The I/Q algorithm is known and used mostly in Doppler techniques. The algorithm was selected due to its simplicity and ease of implementation. However, it should be noted here, that the frequency shift can be also estimated by other techniques which often perform better and characterize by higher accuracy, for example energy-balanced [38] or correlation based [19], [24], [39].

\section{CONClusions}

A new method for determination of ultrasound attenuation coefficient has been presented. The method is a modification of the common attenuation estimation from the downshift of the mean frequency of the propagating pulse. The assumptions are the same: a Gaussian spectrum of the scanning pulse and medium attenuation varying linearly with frequency. In practice, the diffraction phenomenon and noise significantly affect the frequency estimate and consequently the value of the determined attenuation. However, these errors can be reduced by band-pass filtering with properly selected cutoff frequencies. The proposed method removes the disrupted portion of the spectrum of the received signal and uses the filtered data for the calculation of the mean frequency of the spectrum. To this end a new equation was derived, describing the relation between the pulse spectrum shift and pulse spectral width, tissue attenuation and frequency limits.

The method has been examined using simulated and experimental data. Simulation results revealed the usefulness of the new equation for the mean frequency calculation from the limited Gaussian spectrum. In some cases, this mean frequency differed significantly from the frequency determined from nonfiltered data. The measurements were performed using a single circular transducer and a tissue phantom. Phantom experiments indicated that the estimate errors caused by the beam diffraction and noise could be reduced by proper band-pass filtering. The improved accuracy of the attenuation estimate was shown. The proposed method is especially useful for processing data from echoes returned from a large depth, where the SNR is low and distortion of the spectrum due to beam diffraction is relatively large.

\section{ACKNOWLEDGMENT}

This work has been supported by Polish National Science Centre (grant 2011/01/B/ST7/06728) and by the European Regional Development Fund under the Innovative Economy Operational Programme (grant POIG.01.03.01-14-012/08-00)

\section{REFERENCES}

[1] G. Treece, R. Prager, and A. Gee, "Ultrasound attenuation measurement in the presence of scatterer variation for reduction of shadowing and enhancement", IEEE Trans. Ultrason. Ferroelectr. Freq. Control, vol. 111, no. 5, pp. 2346-60, 2005.

[2] M. L. Oelze, and W. D. O'Brien, Jr., "Frequency-dependent attenuationcompensation functions for ultrasonic signals backscattered from random media", The Journal of Acoustical Society of America vol. 123, no. 3, pp. 2308-2319, 2002 .

[3] B. J. Oosterveld, J. M. Thijssen, P. C. Hartman, R. L. Romijn, and G. J. Rosenbusch, "Ultrasound attenuation and texture analysis of diffuse liver disease: methods and preliminary results", Phys. Med. Biol., vol. 36, no. 8, 1039-1064, 1991.

[4] Z. F. Lu J. A. Zagzebski, and F. T. Lee, "Ultrasound Backscatter and Attenuation in Human Liver With Diffuse Disease", Ultrasound in Med. \& Biol. vol. 25, no. 7, pp. 1047-1054, 1999.

[5] K. Nam , J. A. Zagzebski, and T. J. Hall, "Quantitative Assessment of In Vivo Breast Masses Using Ultrasound Attenuation and Backscatter", Ultrasonic Imaging vol. 35, no. 2, pp. 146-161, 2013.

[6] Y. Saijo, "High Frequency Acoustic Properties of Tumor Tissue" in Ultrasonic Tissue Characterization, Springer Japan, 1996, ch. 12, pp. 217-229.

[7] T. A. Bigelow, B. L. McFarlin, W. D. O’Brien, Jr., and M. L. Oelze, "In vivo ultrasonic attenuation slope estimates for detection cervical ripening in rats: Preliminary results", The Journal of Acoustical Society of America vol. 123 , no. 3, pp. 1794-1800, 2008

[8] B. L. McFarlin, T. A. Bigelow, Y. Laybed, W. D. O'Brien, M. L. Oelze, and J. S. Abramowicz, "Ultrasonic attenuation estimation of the pregnant cervix: a preliminary results", Ultrasound in Obstetrics and Gynecology, vol. 36, pp. 218-225, 2010. 
[9] Y. Labyed, T. A. Bigelow, and B. L. McFarlin, "Estimate of the attenuation coefficient using a clinical array transducer for the detection of cervical ripening in human pregnancy", Ultrasonics vol. 51, pp. 34-39, 2011.

[10] B. L. McFarlin, J. Balash, V. Kumar, T. A. Bigelow, X. Pombar, J. S. Abramowicz, and W. D. O'Brien, Jr., "Development of an Ultrasonic method to Detect Cervical Remodeling In Vivo in Full-Term Pregnant Women", Ultrasound in Med. \& Biol., vol. 41, no. 9, pp. 2533-2539, 2015.

[11] B. L. McFarlin, V. Kumar, T. A. Bigelow, D. G. Simpson, R. C. WhiteTraut, J. S. Abramowicz, and W. D. JrO'Brien, Jr., "Beyond Cervical Length: A Pilot Study of Ultrasonic Attenuation for Early Detection of Preterm Birth Risk", Ultrasound in Med. \& Biol., vol. 41, no. 11, pp. 3023-3029, 2015.

[12] H. Piotrzkowska, J. Litniewski, E. Szymanska, and A. Nowicki, "Quantitative sonography of basal cell carcinoma", Ultrasound in Med. \& Biol., vol. 41, no. 3, pp. 748-759, 2015.

[13] H. J. Nieminen, S. Saarakkala, M. S. Laasanen, J. Hirvonen, J. S Jurvelin, and J. Töyräs , "Ultrasound Attenuation in Normal and Spontaneously Degenerated Articular Cartilage", Ultrasound in Med. \& Biol., vol. 30, no. 4, pp. 493-500, 2004.

[14] A. E. Worthington and M. D. Sherar, "Changes in UltrasoundProperties of Porcine Kidney Tissue During Heating", Ultrasound in Med. \& Biol., vol. 27, no. 5, pp. 673-682, 2001.

[15] V. Zderic, A. Keshavarzi, M. A. Andrew, S. Vaezy, and R. W. Martin, "Attenuation of Porcine Tissues In Vivo After High Intensity Ultrasound Treatment", Ultrasound in Med. \& Biol., vol. 30, no. 1, 61-66, 2004.

[16] J. Kemmerer, S. Chang, and M. Oelze, "A New Approach for Detecting Attenuation Changes During High-Intensity Focused Ultrasound", Proceedings of the 2010 IEEE Ultrasonics Symposium (IUS), pp. 1404-1407, 2010.

[17] R. Kuc, M. Schwartz, and L. Von Micsky, "Parametric Estimation of the Acoustic Attenuation Coefficient Slope for Soft Tissue", Proceedings of the 1975 IEEE Ultrasonics Symposium (IUS), pp. 44-47, 1976.

[18] P. A. Narayana and J. Ophir, "On the validity of the linear approximation in the parametric measurement of attenuation in tissues", Ultrasound in Med. \& Biol. vol. 9, no. 4, pp. 357-361, 1983.

[19] H. Kim and T. Varghese, "Attenuation estimation using spectral crosscorrelation", IEEE Trans. Ultrason. Ferroelectr. Freq. Control vol. 54, no. 3, 510-519, 2007

[20] R. Kuc and M. Schwartz, "Estimating the acoustic attenuation coefficient slope for liver from reflected ultrasound signals", IEEE Trans. Sonics Ultrason. vol. 26, no. 5, pp. 353-361, 1979.

[21] M. Insana, J. Zagzebski, and E. Madsen. , "Improvements in the Spectral Difference Method for Measuring Ultrasonic Attenuation", Ultrasonic Imaging vol. 5, no. 4, 331-345, 1983.

[22] R. Kuc, "Estimating acoustic attenuation from reflected ultrasound signals: Comparison of spectral-shift and spectral-difference approaches", IEEE Trans. Acoust., Speech, Signal Process. vol. 32, no. 1, pp. 1-6, 1984.

[23] L. X. Yao, J. A. Zagzebski, and E. L. Madsen, "Backscatter coefficient measurements using a refernce phantom to extract depth-dependent instrumentation factors", Ultrasonic Imaging vol. 12, no. 1, pp. 58-70, 1990.

[24] H. Kim and T. Varghese, "Hybrid spectral domain method for attenuation slope estimation”, Ultrasound in Med. \& Biol., vol. 34, no. 11, 1808-1819, 2008.

[25] Y. Labyed and T. A. Bigelow, "A theoretical comparison of attenuation measurement techniques from backscattered ultrasound echoes", The Journal of the Acoustical Society of America, vol. 129, pp. 2316-2324, 2011.

[26] T. A. Bigelow and Y. Labyed, "Attenuation Compensation and Estimation, in Quantitative Ultrasound in Soft Tissues", editors: Mamou J., Oelze M. L., Springer 2013.

[27] E. Omari, H. Lee, and T. Varghese, "Theoretical and phantom based investigation of the impact of sound speed and backscatter variations on attenuation slope estimation", Ultrasonics vol. 51, no. 6, pp. 758-767, 2011.

[28] K. Samimi and T. Varghese, "Performance Evaluation of the Spectral Centroid Downshift Method for Attenuation Estimation", IEEE Trans. Ultrason. Ferroelectr. Freq. Control vol. 62, no. 5, pp. 871-880, 2015.

[29] R. D. Willink and D. H. Evans, "The Effect of Noise and High-Pass Filtering on the Estimation of Mean Blood Velocity Using Wide and Narrow Ultrasound Beams", IEEE Trans. Biomed. Eng. vol. 43, no. 3, 229-237, 1996.

[30] E. P. Papadakis, "Correction for Diffraction Losses in the Ultrasonic Filed of a Piston Source', The Journal of Acoustical Society of America vol. 31 , no. 2 , pp. 150-152, 1959 .
[31] Z. Klimonda J. Litniewski, and A. Nowicki, "Correcting for bounded bandwidth when estimating tissue attenuation from mean frequency downshift", Proceedings of the 2012 IEEE Ultrasonics Symposium (IUS), 2012.

[32] T. L. Szabo, "Diagnostic Ultrasound Imaging: Inside Out", Elsevier Academic Press, 2004

[33] P. H. Rogers and A. L. Van Buren, "An exact expression for the Lommel diffraction correction integral", The Journal of Acoustical Society of America vol. 55, no. 4, pp. 724-728, 1974.

[34] T. A. Bigelow, B. L. McFarlin, and W. D. O'Brien Jr., "Correcting for Focusing when Estimating Attenuation for Tissue Characterization based on Gaussian Approximations of the Beam Profie", Proceedings of the 2007 IEEE Ultrasonics Symposium (IUS), pp. 2497-2500, 2007.

[35] R. F. Wagner, S. W. Smith, J. M. Sandrik, and H. Lopez "Statistics of speckle in ultrasound b-scans", IEEE Trans. Ultrason. Ferroelectr. Freq. Control vol. 30, no. 3, 156-163, 1983.

[36] W. D. Barber, J. W. Eberhard, S. G. Karr, "A new time domain technique for velocity measurement using Doppler ultrasound", IEEE Trans. Biomed. Eng. vol. BME-32, no. 3, 213-229, 1985.

[37] D. H. Evans and W. N. McDicken, "Doppler Ultrasound: Physics, Instrumentation and Signal Processing”, John Wiley \& Sons Ltd., 2000.

[38] R. Bamler, "Doppler frequency estimation and the Cramér-Rao bound", IEEE Trans. Geosci. Remote Sens. vol. 29, no. 3, 385-390, 1991.

[39] K. Samimi and T. Varghese, "Optimum diffraction-corrected frequencyshift estimator of the ultrasonic attenuation coefficient", IEEE Trans. Ultrason. Ferroelectr. Freq. Control vol. 63, no. 5, 691-702, 2016.

[40] N. Golyandina, V. Nekrutkin, and A. A. Zhigljavsky, "Analysis of time Series Structure: SSA and related techniques", Chapman \& Hall/CRC, 2001

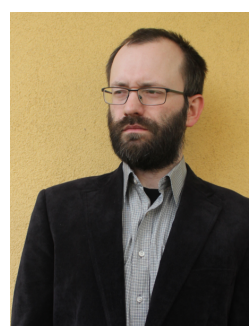

Ziemowit Klimonda graduated from the Faculty of Physics, Warsaw University, Poland, in 2002. Since 2002 he has been employed at the Institute of Fundamental Technological Research, Polish Academy of Sciences, where he received a Ph.D in 2013. His research interest are ultrasound imaging, signal processing, parametric imaging and synthetic aperture techniques.

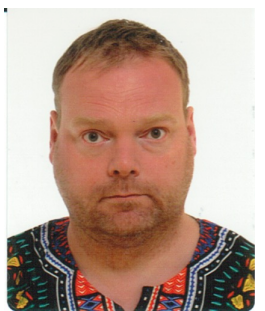

Michiel Postema (A'01-S'02-M'05-SM'08) received an M.Sc. degree in geophysics from Utrecht University, The Netherlands, in 1996 and a Doctorate in the physics of fluids from the University of Twente, Enschede, The Netherlands, in 2004. Following a post-doctoral position at Ruhr-Universität Bochum, Germany, between 2005 and 2007, he became Lecturer in Engineering at The University of Hull, England. He was granted an Emmy Noether Research Group at Ruhr-Universität Bochum in 2009 and a Visiting Professorship at the University of Orléans, France, in 2010. In the same year, he obtained the Chair in Experimental Acoustics at the University of Bergen, Norway. He has been Visiting Professor in Electrical Engineering at the University of the Witwatersrand, Johannesburg, South Africa, since 2015, and Professor and Head of the Department of Ultrasound at the Institute of Fundamental Technological Research of the Polish Academy of Sciences since 2016. Professor Dr. Postema is Associate Editor of Applied Acoustics (Elsevier), the IEEE Transactions on Ultrasonics, Ferroelectrics, and Frequency Control (IEEE), and Ultrasonics (Elsevier). His particular expertise lies in analyzing medical microbubble behavior under sonication and in high-speed photography. $\mathrm{He}$ also explores non-medical applications of bubbles and droplets in sound fields. 


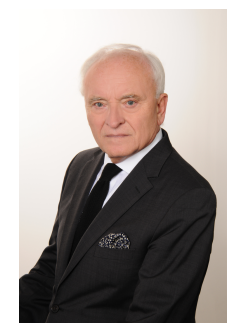

Andrzej Nowicki graduated from the Dept. of Electronics, Warsaw University of Technology in 1969.

$\mathrm{He}$ received his Ph.D. and D.Sc. from Institute of Fundamental Technological Research IFTR, Polish Academy of Sciences in 1976 and 1980. He became a full Professor in 1992. From 1995 till 2013 he was Director for Research and Director of IFTR. In 1972 he conducted research in the field of ultrasound at CNRS, Paris, France. In 1978 and later in1981 he joined a research team at the Institute of Applied Physiology and Medicine, Seattle, WA, USA. In 1986 and 1997 he worked at Drexel University, Philadelphia, PA, USA, being involved in breast Doppler imaging and also being responsible for teaching graduate courses in Advanced Ultrasound. His research interests include pulsed Doppler, cardiac imaging, transcranial and tissue flow Doppler, high frequency ultrasonic imaging, speckles statistics in tumor diagnostics and flow mediated dilation. He was awarded with the Pioneer Award from AIUM in 1988 and became a Honorary Fellow of AIUM in 1990. He was President of The Polish Ultrasound Society 2002-2008.

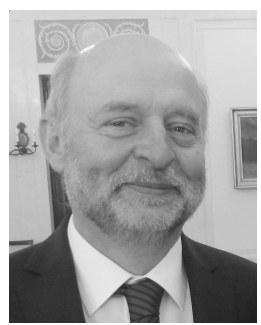

Jerzy Litniewski is a Professor at the Polish Academy of Science heading the Bioacoustics Laboratory in the Institute of Fundamental Technological Research. He received M.Sc. degree from Warsaw University in Physics, Ph.D. degree in scanning acoustic microscopy and D.Sc. degree in bone sonography from the Polish Academy of Science. His research interests involve the many areas of ultrasound-tissue interaction, including soft and bone tissue characterization, acoustic microscopy, in vivo imaging of tissue attenuation and quantitative sonography of breast and skin lesions. 